\title{
A ESPIRITUALIDADE NA EDUCAÇÃO MUSICAL E O PROCESSO DE APRENDIZAGEM: UMA REVISÃO INTEGRATIVA
}

\author{
Spirituality in music education and the \\ learning process: an integrative review
}

\author{
Espiritualidad en la educación musical y \\ el proceso de aprendizaje: una revisión \\ integrativa
}

\author{
Denise Maria Bezerra \\ Universidade Federal de Santa Catarina \\ denisebezerra9@hotmail.com \\ Francisco Antonio Pereira Fialho \\ Universidade Federal de Santa Catarina \\ fapfialho@gmail.com
}

\begin{abstract}
Resumo: A pesquisa científica da espiritualidade na área da música é relativamente recente e ainda pouco explorada no que se refere à sua relação com o processo de aprendizagem. Este trabalho teve como objetivo fazer uma revisão integrativa da literatura, buscando estudos que investiguem a influência da espiritualidade no processo de aprendizagem musical, em nove bases de dados. A revisão seguiu as diretrizes do protocolo PRISMA e foi complementada pelo modelo SPIDER. Na primeira etapa foram selecionados 25 artigos considerados relevantes e na etapa seguinte, 11 artigos incluídos para análise. Os resultados indicaram que a espiritualidade no processo de aprendizagem musical promove o bem-estar e incentiva a conectividade entre os alunos e professores, além de favorecer a criatividade e o desenvolvimento de habilidades e estratégias de aprendizagem musical. A principal contribuição do trabalho revela que não são as técnicas que promovem a espiritualidade, mas o estado de fluxo que ocorre no processo.
\end{abstract}

Palavras-chave: Espiritualidade. Educação musical. Processo de aprendizagem.

\begin{abstract}
The scientific research on spirituality in the field of music is relatively recent and still little explored regarding its relation with the learning process. This work aimed to make an integrative literature review, seeking studies that investigate the influence of spirituality in the process of musical learning in nine databases. The review followed the PRISMA protocol guidelines and was complemented by the SPIDER model. In the first stage, 25 articles considered relevant were selected and in the next stage, eleven articles included for analysis. The results indicated that spirituality in the music learning process promotes wellbeing and encourages connectivity between students and teachers, as well as fostering creativity and the development of musical learning skills and strategies. The main contribution of the work reveals that it is not the techniques that promote spirituality, but the flow state that occurs in the process.
\end{abstract}

Keywords: Spirituality. Music education. Learning process.

Resumen: La investigación científica sobre espiritualidad en el área de la Música es relativamente reciente y aún poco explorada en cuanto a su relación con el proceso de aprendizaje. Este trabajo tuvo como objetivo realizar una revisión integrativa de la literatura, buscando estudios que investiguen la influencia de la espiritualidad en el proceso de aprendizaje musical, en nueve bases de datos. La revisión siguió las pautas del protocolo PRISMA y se complementó con el modelo SPIDER. En la primera etapa se consideraron relevantes 25 artículos y en la siguiente etapa se incluyeron 11 artículos para su análisis. Los resultados indicaron que la espiritualidad en el proceso de aprendizaje musical promueve el bienestar y fomenta la conectividad entre estudiantes y docentes, además de promover la creatividad y el desarrollo de habilidades y estrategias de aprendizaje musical. La principal contribución del trabajo revela que no son las técnicas las que promueven la espiritualidad, sino el estado de flujo que se produce en el proceso.

Palabras clave: Espiritualidad. Educación musical. Proceso de aprendizaje. 


\section{INTRODUÇÃO}

A temática da espiritualidade é ainda cercada de tabus na pesquisa científica. Em razão da herança positivista que tem suas bases no empirismo inglês do século XVIII, a dimensão espiritual foi excluída da bancada científica, quando passou a predominar o pensamento reducionista. A razão e a percepção pelos sentidos passaram a ser consideradas as únicas faculdades capazes de dar acesso a um conhecimento verdadeiro (Sommerman, 2008). A espiritualidade foi relegada ao patamar não científico, pois o ceticismo metafísico afirma a "impossibilidade de um conhecimento que não seja sensível e mensurável” (Sommerman, 2008, p. 18).

Nas últimas décadas, esse cenário vem se modificando. O constructo "espiritualidade" tem sido objeto de crescente interesse por pesquisadores das áreas de administração (Tecchio; Cunha; Brand, 2018; Tecchio; Cunha; Santos, 2016), da saúde (Draper, 2012), da educação (Strieder; Herbert, 2017), das neurociências (Bouso; González; Fondevila; Cutchet; Fernández; Barbosa; Alcázar-Córcoles; Araújo; Barbanoj; Fábregas; Riba, 2012; Bouso; Palhano-Fontes; Rodríguez-Fornells; Ribeiro; Sanches; Crippa; Hallak; Araujo; Riba, 2015; Clonigert; Svrakic; Przybeck, 1993; Mccrae, 2009).

Dessa forma, pretende-se compreender como a espiritualidade vem sendo utilizada na área da música, no campo da educação musical. Tendo em vista a abrangência do termo "educação musical", propõe-se delimitar o escopo desta pesquisa aos processos de aprendizagem musical. Nesse contexto, a aprendizagem refere-se ao âmbito da escola regular e aula particular de música, aos ambientes e situações de ensino-aprendizagem instrumental e coral, bem como aos espaços de musicalização de adultos e crianças.

Neste trabalho de revisão integrativa ${ }^{1}$ da literatura, entende-se a espiritualidade como um aspecto transcendente do indivíduo, logo o termo "espiritualidade" é considerado similar ao termo "transcendência". O objetivo desta revisão é conhecer de que forma está sendo abordada a espiritualidade na área da educação musical, e em especial, nos processos de aprendizagem. Mais especificamente, pretende-se responder: como a espiritualidade está presente na educação musical e de que forma se relaciona aos processos de aprendizagem musical?

O referencial da metodologia empregada é sustentado por Whittemore e Knafl (2005), Abad-Corpa, Gonzalez-Gil, Martínez-Hernández, Barderas-Manchado, Cuesta-Benjumea, Monistrol-Ruano e Mahtani-Chugani (2012), Cooke, Smith e Booth (2012), Botelho, Cunha e Macedo (2011). As fontes de busca foram as bases de dados Scielo, Education Resources Information Center (ERIC); Scopus, PsycInfo, Redalyc, EBSCO, Web of Science, Science Direct

\footnotetext{
1 A revisão integrativa é a mais ampla abordagem metodológica referente às revisões, permitindo a inclusão de estudos experimentais e não experimentais para uma compreensão completa do fenômeno analisado (Souza; Silva; Carvalho, 2010, p. 103).
} 
e Portal Capes. Os trabalhos elegiveis para a análise nesta revisão foram os de abordagem qualitativa, sem restrição de país de origem, nos idiomas inglês, português e espanhol e publicados nos últimos cinco anos.

Como se trata de processos de aprendizagem concentrados na área da educação musical, os participantes consistiram em estudantes e professores de música, basicamente. No entanto, experiências vividas descritas em autoetnografias foram também incluídas, por relatarem situações de aprendizagem musical do pesquisador enquanto sujeito da pesquisa, relacionadas aos aspectos espirituais - ou de transcendência.

\section{REFERENCIAL TEÓRICO}

$\mathrm{Na}$ abordagem da psicologia positiva, a espiritualidade está inserida no grupo da transcendência e é considerada uma das 24 forças de caráter. Nesse contexto, espiritualidade significa "possuir crenças coerentes sobre o propósito superior e o significado do universo; saber onde alguém se encaixa no esquema maior; possuir crenças sobre o sentido da vida que moldam a conduta e proporcionam conforto"2 (Park; Peterson; Seligman, 2004, p. 606, tradução nossa). Pesquisadores admitem as experiências transcendentes em música relacionadas à espiritualidade, chegando a considerar a dimensão do sagrado (Fung, 2017), ou ainda a interação de música e religião (Demmrich, 2020).

\section{Estado de fluxo/platô e experiências de pico}

Uma teoria bastante aceita entre os pesquisadores da área da psicologia da música e educação musical e que estuda a experiência de transcendência é a teoria do fluxo (flow), do psicólogo húngaro Mihaly Csikszentmihalyi (2008). Nessa perspectiva, os pesquisadores Ascenso, Williamon e Perkins (2017) advertem que esta temática foi destacada nos primeiros debates humanísticos (por exemplo, com Maslow, a partir de 1954), mas desde então tem sido largamente negligenciada, devido à falta de evidências empíricas e a um enfoque predominante no prazer ou experiência hedônica. Lembram que a área do bem-estar do músico tem recebido menos atenção nessa modalidade de investigação, com exceção das pesquisas relacionadas ao estado de fluxo (flow), e que artistas e atletas são particularmente propensos a alcançar tais experiências (Ascenso; Williamon; Perkins, 2017).

Entende-se como estado de fluxo uma situação de grande envolvimento e prazer, de profunda concentração e perfeita integração. Para Csikszentmihalyi (2008), nesses casos, a energia de um indivíduo estaria concentrada na experiência, de modo que desaparecem pensamentos e sentimentos contraditórios, a noção de tempo se esvai e as horas parecem passar como minutos.

\footnotetext{
2 No original: "Having coherent beliefs about the higher purpose and meaning of the universe; knowing where one fits within the larger scheme; having beliefs about the meaning of life that shape conduct and provide comfort."
} 
De acordo com Bezerra (2019), os mesmos elementos da experiência de fluxo descrita por Csikzentmihalyi já haviam sido definidos pelo psicólogo americano Abraham Harold Maslow como "experiência de platô". Em 1964, Maslow publica Religions, values and peak experiences, e sustenta que todas as pessoas podem ter experiências de pico e experiências de platô. Nas palavras do autor:

A própria experiência de pico muitas vezes pode significativamente ser chamada de "pequena morte", e um renascimento em vários sentidos. Quanto menos intensa a experiência platô, é mais frequentemente experimentada como puro prazer e felicidade, como, digamos, em uma mãe sentada em silêncio olhando, por horas, o seu bebê brincando, maravilhada, pensando, filosofando, sem acreditar muito no que vê. Ela pode experimentar isso como uma experiência contemplativa muito agradável e contínua, e não como algo semelhante a uma explosão do clímax que então termina (Maslow, 1964, p. 4, tradução nossa).

No entanto, Maslow constata que há pessoas que não estão interessadas ou até mesmo fogem dessas experiências:

Eu finalmente comecei a usar a palavra "non-peaker" para descrever não a pessoa que é incapaz de ter experiências de pico, mas sim a pessoa que tem medo delas, que as reprime, que as nega, que se afasta delas, ou que "esquece" delas. Minhas investigações preliminares sobre as razões dessas reações negativas às experiências de pico me levaram a algumas impressões (não confirmadas) sobre por que certos tipos de pessoas renunciam a suas experiências de pico (Maslow, 1964, p. 12, tradução nossa). ${ }^{3}$

Por essa razão, o autor postula que essa visão da vida leva a pessoa a considerar suas experiências transcendentes como algum tipo de insanidade e tem medo de ser dominada pelas emoções, o que pode interpretar como perda do controle mental (Maslow, 1964).

Interessado na relação de música e experiências de pico, Maslow publica em 1968 um artigo no periódico Music Educators Journal, com o título "Music education and peak experiences":

Quero relatar que as duas maneiras mais fáceis de obter experiências de pico (em termos de estatísticas simples em relató-

\footnotetext{
3 No original: "I finally began to use the word 'non-peaker' to describe, not the person who is unable to have peak-experiences, but rather the person who is afraid of them, who suppresses them, who denies them, who turns away from them, or who 'forgets' them. My preliminary investigations of the reasons for these negative reactions to peak-experiences have led me to some (unconfirmed) impressions about why certain kinds of people renounce their peak-experiences."
} 
rios empíricos) são através da música e do sexo. Vou deixar de lado a educação sexual, pois essas discussões são prematuras - embora eu tenha certeza de que um dia não vamos rir disso, mas levaremos isso muito a sério e ensinaremos às crianças que gostam de música, como o amor, como um insight, como um belo prado, como um bebê fofo, ou qualquer outra coisa, que existem muitos caminhos para o céu, e sexo é um deles, e a música é um deles. Estes são os mais fáceis, os mais difundidos, e os mais fáceis de entender (Maslow, 1968, p. 167, tradução nossa). ${ }^{4}$

Tal postura diante das experiências transcendentes nos oferece uma pista a respeito do reduzido interesse dos pesquisadores em relação a essa temática na área da psicologia da música, pois vem ao encontro daquilo que Maslow apontou como a exclusão do sagrado e do transcendente da jurisdição da ciência. Para esse autor, isso impossibilita o estudo, em princípio, de "certos aspectos do abstrato: psicoterapia, experiência religiosa naturalista, criatividade, simbolismo, jogo, teoria do amor, experiências místicas e de ponta, para não mencionar poesia, arte e muito mais"5 (Maslow, 1970, p. 9-10, tradução nossa).

Nesse sentido, Maslow define a transcendência e a dimensão espiritual como o topo da pirâmide na hierarquia das necessidades no desenvolvimento humano, para além de alcançar a autorrealização. O autor sustenta que a autotranscendência leva o indivíduo a encontrar "valores espirituais", que denominou "valores do ser" (Maslow, 1971). Ressalta que a constante expansão de consciência, por meio da experiência de platô (fluxo) e experiências culminantes, leva ao processo de "autoatualização", que no referencial teórico de Maslow corresponde ao termo "individuação".

De igual modo, o compositor e professor de música americano Anthony John Palmer (2006) destaca que os professores de música devem buscar o que existe de musicalidade nos materiais utilizados, para garantir exercícios de habilidade e qualidade no processo de aprendizagem. O autor lembra que uma forma criativa de ensino possui potencial para alcançar um nível de consciência espiritual, ou seja, que transcenda a um nivel maior de existência. Palmer defende que "aprender e cultivar tais possibilidades desde cedo

\footnotetext{
4 No original: "I want to report that the two easiest ways of getting peak experiences (in terms of simple statistics in empirical reports) are through music and through sex. I will push aside sex education, as such discussions are premature - although I am certain that one day we will not giggle over it, but will take it quite seriously and teach children that like music, like love, like insight, like a beautiful meadow, like a cute baby, or whatever, that there are many paths to heaven, and sex is one of them, and music is one of them. These happen to be the easiest ones, the most widespread, and the ones that are easiest to understand."

5 No original: "This makes impossible in principle the study, for instance, of certain aspects of the abstract: psychotherapy, naturalistic religious experience, creativity, symbolism, play, the theory of love, mystical and peak-experiences, not to mention poetry, art, and a lot more."

6 Termo original: "being-values".
} 
aumentaria as chances de que a autoatualização pudesse ocorrer"7 (Palmer, 2006, p. 148, tradução nossa).

Assim, o processo de aprendizagem musical e sua relação com a espiritualidade é uma questão central nesta revisão. A palavra "processo" remete a algo ainda não acabado, em construção, em desenvolvimento. Processo é uma palavra que deriva do latim processus, projeção, descende de pro, para frente, e cedere, ir; está relacionada com percurso, e significa "avançar" ou "caminhar para frente". Interessa, pois, observar o avanço, o caminhar para frente na musicalização ou no trabalho com uma obra musical em fase de estudo ou de manutenção, durante a qual ocorrem com o estudante de música mudanças em diversos niveis do ser (Bezerra, 2016, 2019). O aluno, aprendente musical, pode transcender os limites da técnica e da transmissão do conteúdo musical por parte do professor, e incorporar novas modalidades de conexão interior. Maslow denominou essa conexão de "motivação intrínseca", que proporciona estados mentais de platô e podem levar a experiências culminantes (peak experiences) (Maslow, 1971, p. 174).

\section{MÉTODOS}

\section{Coleta de dados}

Os dados foram coletados a partir da busca em nove bases. Como critérios de inclusão, foram selecionados apenas artigos revisados por pares, em idioma inglês, português e espanhol, no período dos últimos cinco anos, (desde 2014, incluindo os primeiros meses de 2019).

A busca realizou-se no período de junho a agosto de 2019 nas bases de dados Scielo, Education Resources Information Center (ERIC); Scopus, PsycInfo, Redalyc, EBSCO, Web of Science, Science Direct e Portal Capes. A base Scielo foi utilizada por abranger uma coleção selecionada de periódicos científicos brasileiros. A Scopus, por ser a maior base de dados bibliográfica internacional nas áreas de ciência, tecnologia, medicina, ciências sociais e artes e humanidades. A Web of Science é uma base multidisciplinar, de atualização semanal, que inclui periódicos relacionados à área de estudo desta pesquisa, tal qual a base Science Direct. A Redalyc (Rede de Revistas Científicas da América Latina e Caribe, Espanha e Portugal) é uma base de dados bibliográfica e de uma biblioteca digital de revistas de acesso aberto, suportado pela Universidade Autónoma do Estado do México com a ajuda de numerosas outras instituições de ensino superior e dos sistemas de informação. Considera-se imprescindivel para esta pesquisa a inclusão dessa base em função do volume de estudos realizados em língua portuguesa e espanhola em nossa área de interesse. A base ERIC dá acesso aos trabalhos relacionados

\footnotetext{
7 No original: "My argument is that learning of and cultivating such possibilities early on would increase the chances that self-actualization could occur."
} 
à educação musical, fundamental na área de estudo desta pesquisa. A base de dados da Associação Americana de Psicologia (APA), a PsyInfo, foi consultada porque entende-se que um trabalho que lida com comportamento humano deve ter sua busca em bases relacionadas à psicologia. A EBSCO, por ser uma plataforma que permite a busca em diversas bases de dados disponíveis também via Portal Capes. Esta última serviu também como uma base de referência para observar o volume total de estudos somados nas bases indexadas.

Os estudos foram elegiveis a partir das orientações contidas no mnemônico SPIDER, proposto por Cooke, Smith e Booth (2012), da seguinte forma:

S - sample (amostra): "spirituality" AND "music education" AND "learning process". Nesta revisão foram incluidos estudos sobre a presença da espiritualidade em processos de aprendizagem.

PI - phenomenon of interest (intervenção/fenômenos de interesse): os estudos incluídos quando apresentaram intervenções em aula de música que considerem a presença da espiritualidade. Estudos realizados em práticas pedagógicas e psicopedagógicas no ensino em diferentes espaços de aprendizagem musical que contenham intervenções em grupo que envolvam técnicas tais como mindfulness, imaginação ativa, situações de estado de fluxo (flow), visualização criativa, experiências culminantes. Estudos que descrevam experiências dos professores e alunos considerando a espiritualidade presente no processo de aprendizagem. Estudos que sejam resultados de autoetnografia de individuos que experienciaram situações que envolvem a espiritualidade no processo de aprendizagem musical.

D - design (desenho metodológico): de acordo com Cooke, Smith e Booth (2012), os detalhes do desenho do estudo ajudam a tomar decisões sobre a robustez do estudo e análise. Portanto, foram incluídos estudos quantitativos e qualitativos, estudos teóricos e revisões.

E - evaluation (avaliação): os principais resultados de interesse foram: a descrição do fenômeno, a descrição da intervenção e o detalhamento dos resultados. Interessa avaliar o papel da espiritualidade nos processos e o grau de envolvimento dos indivíduos - educador, educando e outros. Os resultados secundários de interesse são a identificação de novas técnicas e procedimentos que possam emergir desses processos de aprendizagem.

$\mathrm{R}$ - research type (tipo de pesquisa): foram mantidos estudos fenomenológicos, teoria fundamentada, estudos etnográficos, autoetnografias e pesquisa-ação, todos sem restrição de país, nos últimos cinco anos, incluindo 2019.

Os artigos pré-selecionados foram agrupados para leitura dos títulos e resumos, quando foram identificados 25 estudos elegiveis. Nessa primeira 
etapa apenas realizou-se a triagem em que foram identificados e excluídos os estudos repetidos nas bases e os que fogem ao escopo delimitado no objetivo desta revisão. No momento seguinte, foi feita a leitura dos resumos de cada estudo, quando restaram 11 artigos altamente relevantes. Com o objetivo de caracterizar a descrição do fenômeno, a descrição da intervenção e o detalhamento dos resultados, buscaram-se os textos completos disponiveis para análise (Figura 1).

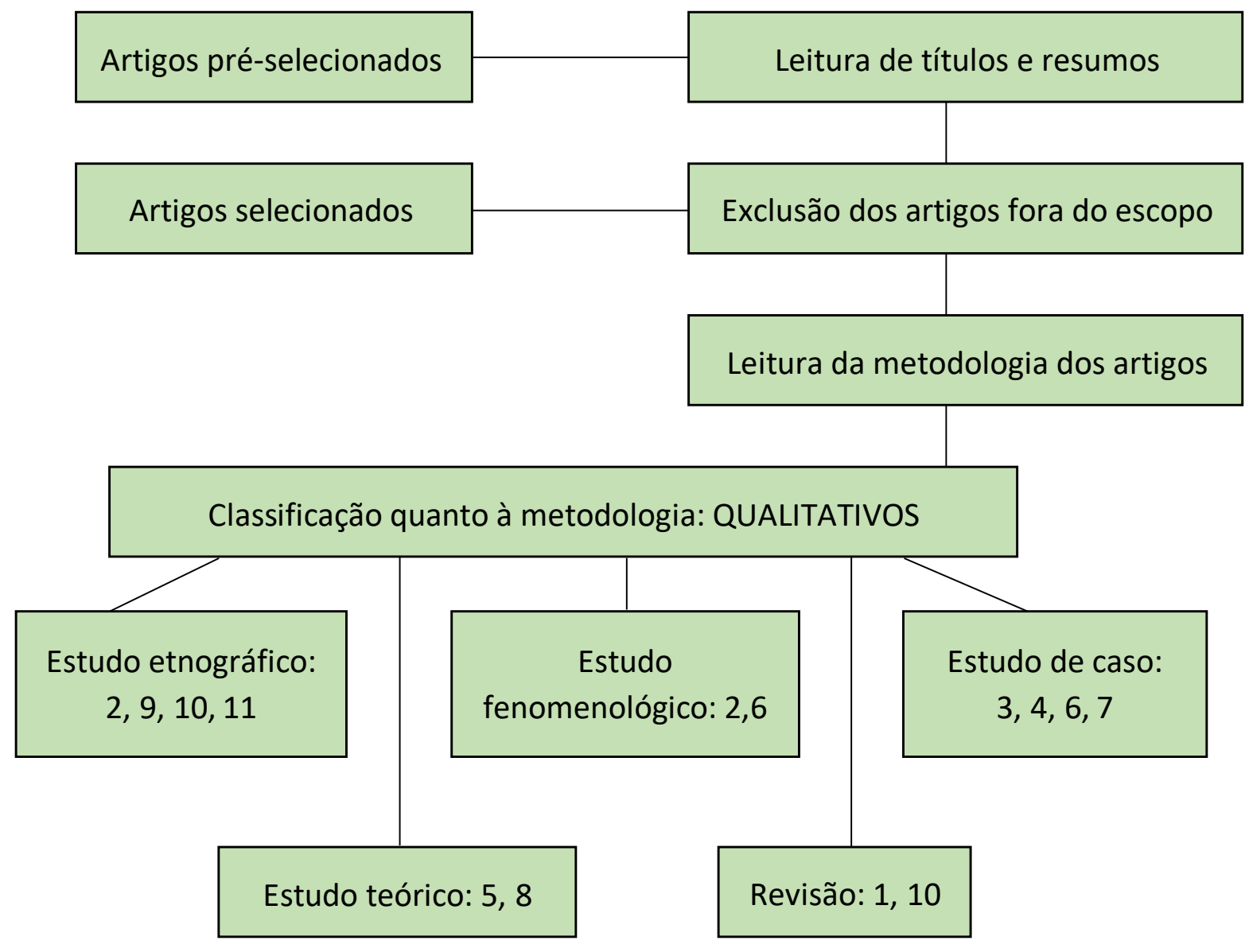

Figura 1: Processo de seleção e análise dos estudos. Fonte: construção dos autores.

\section{RESULTADOS}

$\mathrm{Na}$ amostra final desta revisão, 11 artigos foram considerados altamente relevantes, de acordo com os critérios de inclusão descritos na sessão de métodos. Os trabalhos foram agrupados por base de dados, conforme Quadros 1, 2, 3 e 4. 


\begin{tabular}{|c|c|c|c|}
\hline $\begin{array}{l}\text { Base de } \\
\text { dados }\end{array}$ & Titulo do artigo & Autor & Periódico \\
\hline \multirow{4}{*}{$\begin{array}{l}\text { Portal } \\
\text { Capes }\end{array}$} & $\begin{array}{c}\text { "A conceptual study of spiritua- } \\
\text { lity in selected writings of Émile } \\
\text { Jaques-Dalcroze" }\end{array}$ & $\begin{array}{l}\text { John Habron; } \\
\text { Liesl van Der } \\
\text { Merwe }\end{array}$ & $\begin{array}{l}\text { International } \\
\text { Journal of Music } \\
\text { Education (2017) }\end{array}$ \\
\hline & $\begin{array}{l}\text { "Transmission of araquio music, } \\
\text { songs, and movement conven- } \\
\text { tions: learning, experience, and } \\
\text { meaning in devotional theatre" }\end{array}$ & $\begin{array}{l}\text { Florante P. } \\
\text { Ibarra }\end{array}$ & $\begin{array}{c}\text { Qualitative } \\
\text { Report (2017) }\end{array}$ \\
\hline & $\begin{array}{l}\text { "Adult perspectives of lear- } \\
\text { ning musical instruments" }\end{array}$ & $\begin{array}{l}\text { Kathryn } \\
\text { Roulston; } \\
\text { Peter Jutras; } \\
\text { Seon Joo Kim }\end{array}$ & $\begin{array}{l}\text { International } \\
\text { Journal of Music } \\
\text { Education (2015) }\end{array}$ \\
\hline & $\begin{array}{l}\text { "Becoming music-making music } \\
\text { teachers: connecting music } \\
\text { making, identity, wellbeing, and } \\
\text { teaching for four student teachers" }\end{array}$ & $\begin{array}{l}\text { Kristen } \\
\text { Pellegrino }\end{array}$ & $\begin{array}{l}\text { Research Studies } \\
\text { in Music Educa- } \\
\text { tion (2015) }\end{array}$ \\
\hline
\end{tabular}

Quadro 1: Artigos da base de dados Portal Capes.

\begin{tabular}{|c|c|c|c|}
\hline $\begin{array}{l}\text { Base de } \\
\text { dados }\end{array}$ & Titulo do artigo & Autor & Periódico \\
\hline \multirow{3}{*}{$\begin{array}{l}\text { Web of } \\
\text { Science }\end{array}$} & $\begin{array}{l}\text { "The Dalcroze diamond: a theory } \\
\text { of spiritual experiences in Dal- } \\
\text { croze eurhythmics" }\end{array}$ & $\begin{array}{l}\text { Liesl van Der } \\
\text { Merwe; John } \\
\text { Habron }\end{array}$ & $\begin{array}{l}\text { Music Education } \\
\text { Research (2019) }\end{array}$ \\
\hline & $\begin{array}{l}\text { "Young children and spirituality: } \\
\text { understanding children's connec- } \\
\text { tedness in a group music class" }\end{array}$ & $\begin{array}{l}\text { Elsabe Nortjé; } \\
\text { Liesl van Der } \\
\text { Merwe }\end{array}$ & $\begin{array}{l}\text { International } \\
\text { Journal of Chil- } \\
\text { dren's Spiritua- } \\
\quad \text { lity (2016) }\end{array}$ \\
\hline & $\begin{array}{l}\text { "Mindfulness for singers: the } \\
\text { effects of a targeted mindfulness } \\
\text { course on learning vocal technique" }\end{array}$ & $\begin{array}{l}\text { Anne-Marie L. } \\
\text { Czajkowski; } \\
\text { Alinka E. } \\
\text { Greasley }\end{array}$ & $\begin{array}{l}\text { British Journal } \\
\text { of Music Educa- } \\
\text { tion (2015) }\end{array}$ \\
\hline
\end{tabular}

Quadro 2: Artigos da base de dados Web of Science. 


\begin{tabular}{|c|c|c|c|}
\hline $\begin{array}{c}\text { Base de } \\
\text { dados }\end{array}$ & Titulo do artigo & Autor & Periódico \\
\hline \multirow{2}{*}{ EBSCO } & $\begin{array}{c}\text { "Masterful words: musicianship } \\
\text { and ethics in learning the ney" }\end{array}$ & Banu Senay & $\begin{array}{c}\text { Journal of the } \\
\text { Royal Anthropo- } \\
\text { logical Institute } \\
\text { (2015) }\end{array}$ \\
\cline { 2 - 4 } & $\begin{array}{c}\text { "A conceptual model of spiritua- } \\
\text { lity in music education" }\end{array}$ & $\begin{array}{c}\text { Liesl van Der } \\
\text { Merwe; } \\
\text { John Habron }\end{array}$ & $\begin{array}{c}\text { Journal of Rese- } \\
\text { arch in Music } \\
\text { Education (2015) }\end{array}$ \\
\hline
\end{tabular}

Quadro 3: Artigos da base de dados EBSCO.

\begin{tabular}{|c|c|c|c|}
\hline $\begin{array}{c}\text { Base de } \\
\text { dados }\end{array}$ & Título do artigo & Autor & Periódico \\
\hline $\begin{array}{c}\text { Education } \\
\text { Resources } \\
\text { Information } \\
\text { Center } \\
\text { (ERIC) }\end{array}$ & $\begin{array}{c}\text { "Satis Coleman - a spiritual } \\
\text { philosophy for } \\
\text { music education" }\end{array}$ & $\begin{array}{c}\text { Daniel J. } \\
\text { Shevock }\end{array}$ & $\begin{array}{c}\text { Music Educators } \\
\text { Journal (2015) }\end{array}$ \\
\cline { 2 - 4 } Scopus & $\begin{array}{c}\text { nographic study of a West African } \\
\text { drum and dance ensemble" }\end{array}$ & $\begin{array}{c}\text { Marissa } \\
\text { Silverman }\end{array}$ & $\begin{array}{c}\text { Research Studies } \\
\text { in Music Educa- } \\
\text { tion (2018) }\end{array}$ \\
\hline
\end{tabular}

Quadro 4: Artigos das bases de dados ERIC e Scopus.

\section{Análise crítica dos estudos incluídos}

Os principais resultados de interesse foram: a descrição do fenômeno, a descrição da intervenção e o detalhamento dos resultados. Interessa avaliar o papel da espiritualidade nos processos e o grau de envolvimento dos indivíduos - educador, educando e outros. Os resultados secundários de interesse são a identificação de novas técnicas e procedimentos que possam emergir desses processos de aprendizagem. Os critérios que nortearam a análise crítica dos artigos obedeceram à diretriz do protocolo. Foram ponderados o rigor e as características de cada estudo, observados nesta sequência:

1. A descrição do fenômeno.

2. A descrição da intervenção.

3. O detalhamento dos resultados.

4. Identificação de novas técnicas. 
A partir dessa sequência, buscou-se reconhecer os temas centrais que melhor respondem ao objetivo proposto nesta revisão. Retomando a questão norteadora "como a espiritualidade está presente na educação musical e de que forma se relaciona aos processos de aprendizagem musical?", foi possível identificar três categorias de estudos científicos que correspondem a esta proposição: (1) o bem-estar físico, emocional e espiritual através das práticas de educação musical relacionadas à espiritualidade, juntamente com a conexão interior e com os outros; (2) a criatividade e habilidade técnica no ensino de música como fator estimulante aos estados relacionados à espiritualidade; (3) o resgate de tradições de prática instrumental em grupo e aprendizagem musical, que incluem a espiritualidade.

\section{DISCUSSÃO}

A partir dos dados obtidos, os resultados demonstraram que a dimensão espiritual no processo de aprendizagem musical proporciona o bem-estar físico, emocional e espiritual do ser humano, trazendo beneficios a alunos e professores, em adultos e crianças. O bem-estar e a conexão interior e com os outros compõem a primeira categoria dentre as três que emergiram como questões centrais na análise dos dados. Alguns beneficios, como sentir-se conectado consigo mesmo e o gerenciamento do estresse são fatores demonstrados pelos estudos (Pellegrino, 2015, p. 175). Em estudantes adultos, são constatados os benefícios que os participantes do estudo atribuem ao seu envolvimento em atividades musicais e desafios enfrentados, tais como a motivação para aprender instrumentos, as metas e as estratégias de aprendizagem que empregam espiritualidade (Roulston; Jutras; Kim, 2015).

Nesse sentido, a teoria desenvolvida por Liesl van der Merwe e John Habron (2019), para elucidar os aspectos espirituais do método de educação musical do suiço Émile Jaques-Dalcroze (1865-1950), propõe que a participação prática nesse método pode nos conectar a nós mesmos e aos outros. Pode ainda facilitar experiências transcendentes, holísticas ou transformadoras (Van der Merwe; Habron, 2019). Os autores utilizaram um diamante como uma metáfora para mostrar suas descobertas, o "diamante de Dalcroze", o resultado de um longo estudo que compreende quatro artigos prévios, dois dos quais incluídos nesta revisão.

O bem-estar espiritual e a conectividade foram notados por crianças com idades entre quatro e cinco anos, que assim relataram sua experiência interior com a música: "a música é a melhor", "meu corpo dança", "a música me leva a lugares", "nós podemos brincar juntos", "eu me sinto melhor" (Nortjé; Merwe, 2016). O holismo, o equilíbrio, a experiência estética e o movimento no tempo, no espaço e na energia emergiram como conceitos centrais nos estudos (Van der Merwe; Habron, 2017).

A descrição das situações características das experiências espirituais nos processos de aprendizagem musical apresentada pelos estudos está de acordo com os indicadores do estado de fluxo e de platô, conforme referido neste trabalho (Maslow, 1968, 1970; Csikszentmihalyi, 2008). 
A segunda categoria identificada nesta revisão refere-se à criatividade no ensino como um fator espiritual que parte do professor. A prática de mindfulness em aulas de canto afetou positivamente as relações entre professor e aluno, e melhorou a concentração e foco nas aulas (Czajkowski; Greasley, 2015).

$\mathrm{Na}$ mesma direção está o trabalho pioneiro desenvolvido pela professora americana Satis Coleman (1878-1961), cuja filosofia de educação musical continha uma característica notadamente espiritual, que valorizava a vida saudável e simples, o silêncio, a natureza, e incluía uma perspectiva da existência de Deus (Shevock, 2015). O autor deste estudo aponta que, a partir de Coleman, o profissional da educação musical pode se beneficiar da compreensão mais profunda do trabalho de professores criativos. Shevock (2015, p. 56, tradução nossa) cita a definição de Parker Palmer para a espiritualidade como "o anseio humano eterno de estar ligado a algo maior que nossos próprios egos", 8 e entende essa ideia como sendo uma lente para examinar a espiritualidade de Coleman, relacionada à educação musical.

Há momentos em que as categorias se interpenetram, mantendo pontos em comum no mesmo estudo. Um exemplo disso é o texto que trata do ensino e aprendizagem do ney, uma flauta de bambu oriental. O autor do estudo não só identifica sensibilidades extramusicais relevantes para se tornar um ney- player habilidoso e afetos comunais entre os participantes do ato de escuta, como identifica também novas e criativas habilidades de audição e compreensão musical. Argumenta que quando ocorrem todas essas combinações, "certas disposições éticas são fomentadas no eu moral dos aprendizes, possibilitando novas formas de se relacionar com os outros e com a cidade" (Senay, 2015 , p. 524, tradução nossa). Novamente resgata-se o fator de conectividade e fatores extramusicais, presentes na primeira categoria, combinados com a criatividade para novas habilidades que promovem um desempenho técnico de alto nivel.

Em outro estudo, a autora apresenta fatores como o engajamento e a satisfação por encontrar sentido e propósito da atividade realizada. Tais fatores confirmam o estado de fluxo, garantido quando os professores estão tão envolvidos quanto os alunos:

Através da produção musical dentro da sala de aula, os participantes desenvolveram sua identidade profissional, percebendo que tipo de professores de música eles queriam se tornar: empolgados com o ensino de música e professores de música que usam música para inspirar os alunos a se empolgarem e se engajarem no aprendizado de música (Pellegrino, 2015, p. 175, tradução nossa). ${ }^{10}$

\footnotetext{
8 No original: "The eternal human yearning to be connected with something larger than our own egos."

9 No original: "Certain ethical dispositions are fostered in learners' moral selves, enabling new ways of relating to others and to the city."

${ }^{10}$ No original: "Through music making inside the classroom, participants developed their professional identity, realizing what kind of music teachers they wanted to become: excited about music and music teaching, and music teachers who use music making to inspire students to be excited and engaged in music learning."
} 
Observe-se que no processo dinâmico de interação das categorias, evidencia-se o favorecimento de um desempenho musical superior, conforme constatado. A criatividade e o bem-estar promovem conectividade e melhoram a relação entre alunos e professores. Nesse ambiente e com esse estado mental de fluxo coletivo, as técnicas e estratégias de estudo parecem romper limites do ensino tradicional. Vale a reiteração da citação do estudo que apresenta a teoria com o diamante de Dalcroze:

Propomos que, se a participação na prática de Dalcroze puder nos conectar a nós mesmos, aos outros, ao ambiente, ao sagrado (aquilo que é feito especial, separado, nutrido, amado, apreciado, respeitado ou considerado importante) ou facilitar experiências transcendentes, holísticas ou transformadoras, então experiências espirituais podem ocorrer. Com essa teoria, esperamos criar um aumento de consciência do potencial espiritual da classe Dalcroze e comunicar a reflexão e o tato pedagógico necessários ao ensinar usando a abordagem Dalcroze (Van der Merwe; Habron, 2019, p. 426, tradução nossa). ${ }^{11}$

A criatividade e o alto nivel de habilidade técnica (peak-performance) são elementos presentes nos estados de platô/fluxo e até mesmo das experiências culminantes, descritas por Maslow. Na abordagem da teoria do fluxo, de Mihaly Csikszentmihalyi (2008), o equilíbrio entre desafio e habilidade pode levar ao estado de fluxo (flow). Entretanto, o desequilíbrio ocorre caso o desafio seja muito grande para a habilidade do aluno. Entretanto, o desequilíbrio ocorre caso o desafio seja muito grande para o nivel de habilidade do aluno. Nessa circunstância, se o desafio for alto demais, levará à ansiedade. Por outro lado, uma habilidade muito elevada para um desafio inferior pode causar tédio. No ensino de música, se o professor oferece uma peça musical muito avançada para a habilidade do aluno, o estado de fluxo fica comprometido, gerando ansiedade no estudante. Da mesma forma, uma obra fácil demais para um aluno adiantado também poderá prejudicar o estado de fluxo e, consequentemente, desencadear o tédio no estudo (Bezerra, 2016). Essa situação no processo de aprendizagem musical deixa clara a necessidade de uma abordagem holística, empática e de conectividade entre professor e aluno.

A terceira e última categoria refere-se ao resgate das tradições de educação musical fora do ensino formal. Um exemplo é o estudo etnográfico (Silverman, 2018) conduzido na universidade norte-americana Montclair State University (New Jersey), com o objetivo de investigar um grupo de dança e percussão de música africana (especificamente a música praticada na África Ocidental). O grupo foi criado pela autora do estudo, motivada pela preocu-

\footnotetext{
${ }^{11}$ No original: "We propose that if participation in Dalcroze practice can connect us to ourselves, others, the environment, the sacred (that which is made special, set apart, nurtured, loved, enjoyed, respected or considered important), or facilitate transcendent, holistic or transformative experiences, then spiritual experiences may occur. With this theory, we hope to create heightened awareness of the spiritual potential in the Dalcroze class and communicate the pedagogical thoughtfulness and tact that are required when teaching using the Dalcroze approach."
} 
pação com o fato de prevalecer o repertório canônico da música ocidental nos programas de educação musical da instituição. O esforço foi para promover a diversidade musical no contexto de sua própria escola de música universitária. Dentre os inúmeros relatos dos 36 integrantes, a participante "Melissa" assim define sua experiência com o grupo:

Não sei se é a música ou a aula de Levin, mas a alegria que sinto ao fazer essa música é extasiante. Quer dizer, convenci minha mãe a ir a uma de nossas aulas. Ela tocou axatse e dançou com o coração [...] Ultimamente, ela não tem estado muito feliz em casa. Mas naquele dia ela experimentou uma felicidade genuína. Sem parar. Ela teve uma explosão. Nós tivemos uma explosão (Silverman, 2018, p. 11, tradução nossa). ${ }^{12}$

Estudo semelhante foi realizado na cidade de Peñaranda, provincia de Nueva Ecija, Filipinas, explorando a natureza holística dos processos de transmissão e aprendizagem da música de araquio e das canções como um ritual de teatro. "Araquio", é um teatro folclórico indígena que sobrevive há mais de cem anos. Conforme o próprio autor,

Como uma tradição oral viva, a aprendizagem intergeracional é encontrada como sendo o produto da transmissão por enculturação que ocorre no araquio e acontece dentro da geração genealógica. Os praticantes, através do significado [não dito] da tradição, têm certos fatores não ditos: unidade de propósito, adesão ancestral, unificação da força tribal e experiências compartilhadas (Ibarra, 2017, p. 1031, tradução nossa). ${ }^{13}$

Entende-se que a busca pela tradição oral de transmissão dos conhecimentos musicais nesses grupos, levando em conta os aspectos transcendentes, corrobora os dados explicitados nas categorias anteriores. Aspectos como transcendência, sinergia grupal, conectividade, identidade, comunidade, compartilhamento de experiências, estão presentes em todos os estudos incluídos nesta revisão.

\section{CONSIDERAÇÕES FINAIS}

Esta revisão teve por objetivo conhecer os trabalhos científicos dos últimos cinco anos da área de educação musical que demonstrem a presença da

\footnotetext{
12 No original: "I don't know if it's the music, or Levin's teaching, but the joy I feel when making this music is ecstatic. I mean, I convinced my mom to come to one of our class sessions. She played the axatse and danced her heart out [...] Lately, she hasn't been super happy at home. But that day, she experienced genuine happiness. Non-stop. She had a blast. We had a blast."

${ }^{13}$ No original: "As a living oral tradition, intergerational learning is found to be product of transmission by enculturation occurring in the araquio and happens within genealogical generation. The practitioners, through the unspoken meaning of the tradition, have certain unspoken factors: unity of purpose, ancestral adhesion, unification of tribal strength, and shared experiences."
} 
espiritualidade. Buscamos, mais detidamente, compreender como a espiritualidade se relaciona a esse processo de aprendizagem musical.

Este estudo demonstrou que a espiritualidade no processo de aprendizagem musical promove o bem-estar de forma ampla, contribui para a conexão interior e com os outros. Vale ressaltar que não se trata da experiência hedônica, da busca pelo prazer no fazer musical, apenas. A dimensão espiritual emerge em situações que incluem a criatividade e habilidade técnica no ensino-aprendizagem de música como fator estimulante aos estados mentais diferenciados, tais como estado de fluxo e experiência culminante. Ademais, o resgate das tradições de prática instrumental em grupo e aprendizagem musical, que incluem a espiritualidade, ocorre fora dos muros acadêmicos e do ensino formal e também se apresenta como evidência nesse estudo.

Um objetivo secundário deste estudo era identificar as técnicas diferenciadas de abordagem espiritual na educação musical e nos processos de aprendizagem. Entretanto, percebeu-se que o que determina que haja uma perspectiva relacionada com a espiritualidade no processo de aprendizagem musical não são as técnicas aplicadas (por exemplo, o mindfulness) em si, mas é a experiência de fluxo que ocorre, fruto da expansão de consciência e conexão interior e com os outros. Tal percepção consiste na maior contribuição deste trabalho para o campo da educação musical.

Esse panorama oferece pistas para uma reflexão profunda da prática docente no campo da educação musical, propiciando um convite à expansão de consciência, em uma dinâmica de trabalho que transcende os limites da técnica. O aporte dos estudos aqui apresentados nos auxilia nessa expansão, no sentido de respaldar o emprego de condutas ainda pouco valorizadas em nosso meio.

Por fim, entendemos que a dimensão espiritual na educação musical - ou o nível do psiquismo que ultrapassa nossos próprios egos, como dito consiste em um estado de consciência diferenciado, em que tudo flui. A presença da espiritualidade na educação musical traz a essa área de conhecimento um resgate importante de componentes que nunca deixaram de ser inerentes ao processo de aprender música. Eis um fecundo campo de investigação para novas propostas no ensino e aprendizagem de música.

\section{REFERENCIAS}

ABAD-CORPA, Eva; GONZALEZ-GIL, Teresa; MARTÍNEZ-HERNÁNDEZ, Antonio; BARDERAS-MANCHADO, Ana M.; CUESTA-BENJUMEA, Carmen de la; MONISTROL-RUANO, Olga; MAHTANI-CHUGANI, Vinita. Caring to achieve the maximum independence possible: a synthesis of qualitative evidence on older adults' adaptation to dependency. Journal of Clinical Nursing, [s. l.], v. 21, n. 21-22, p. 3153-3169, 2012.

ASCENSO, Sarah; WILLIAMON, Aaron; PERKINS, Rosie. Understanding the wellbeing of professional musicians through the lens of Positive Psychology. Psychology of Music, [s. l.], v. 45, n. 1, p. 65-81, 2017. 
BEZERRA, Denise. Maria. Ação pianistica e estados de consciência: uma abordagem integrativa transpessoal. [S. l.]: Universidade do Estado de Santa Catarina, 2016.

A dimensão transcendental na prática pianística: uma abordagem integrativa transpessoal. Revista Científica Multidisciplinar Núcleo do Conhecimento, São Paulo, v. 6, n. 8, p. 148-183, 4 set. 2019.

BOTELHO, Louise Lira Roedel; CUNHA, Cristiano Castro de Almeida; MACEDO, Marcelo. O método da revisão integrativa nos estudos organizacionais. Gestão e Sociedade, Belo Horizonte, v. 5, n. 11, p. 121-136, 2011.

BOUSO, José Carlos; GONZÁLEZ, Débora; FONDEVILA, Sabela; CUTCHET, Marta; FERNÁNDEZ, Xavier; BARBOSA, Paulo César Ribeiro; ALCÁZARCÓRCOLES, Miguel Ángel; ARAÚJO, Wladimyr Sena; BARBANOJ, Manel J.; FÁBREGAS, Josep Maria; RIBA, Jordi. Personality, psychopathology, life attitudes and neuropsychological performance among ritual users of ayahuasca: a longitudinal study. PLoS ONE, [s. l.], v. 7, n. 8, e4242, 2012.

BOUSO, José Carlos; PALHANO-FONTES, Fernanda; RODRÍGUEZFORNELLS, Antoni; RIBEIRO, Sidarta; SANCHES, Rafael; CRIPPA, José Alexandre S.; HALLAK, Jaime E. C.; ARAUJO, Draulio B. de; RIBA, Jordi. Long-term use of psychedelic drugs is associated with differences in brain structure and personality in humans. European Neuropsychopharmacology, [s. l.], v. 25, n. 4, p. 483-492, 2015.

CLONIGER, Claude Robert; SVRAKIC, Dragan M.; PRZYBECK, Thomas R. A psychobiological model of temperament and character. Archives of General Psychiatry, [s. l.], v. 50, n. 12, p. 975-990, 1993.

COOKE, Alison; SMITH, Debbie; BOOTH, Andrew. Beyond PICO: The SPIDER tool for qualitative evidence synthesis. Qualitative Health Research, [s. l.], v. 22, n. 10, p. 1435-1443, 2012.

CSIKSZENTMIHALYI, Mihaly. Flow: the psychology of optimal experience. New York: Harper Collins, 2008.

CZAJKOWSKI, Anne-Marie L.; GREASLEY, Alinka E. Mindfulness for singers: the effects of a targeted mindfulness course on learning vocal technique. British Journal of Music Education, Cambridge, v. 32, n. 2, p. 211-233, 2015.

DEMMRICH, Sarah. Music as a trigger of religious experience: what role does culture play?. Psychology of Music, [s. l.], v. 48, n. 1, p. 35-49, 2020.

DRAPER, Peter. An integrative review of spiritual assessment: Implications for nursing management. Journal of Nursing Management, [s. l.], v. 20, n. 8, p. 970-980, 2012. 
FUNG, Annabela. S. K. Music enables the holistic development and discovery of self: a phenomenological study of two Christian musicians. Psychology of Music, [s. l.], v. 45, n. 3, p. 400-416, 2017.

HABRON, John; VAN DER MERWE, Liesl. A conceptual study of spirituality in selected writings of Émile Jaques-Dalcroze. International Journal of Music Education, [s. l.], v. 35, n. 2, p. 175-188, 2017.

IBARRA, Florante P. Transmission of araquio music, songs, and movement conventions: learning, experience, and meaning in devotional theatre. Qualitative Report, [s. l.], v. 22, n. 4, p. 1031-1049, 2017.

MASLOW, Abraham Harold. Religions, Values, and Peak Experiences. Columbus: Ohio State University Press, 1964.

Music Education and Peak Experiences. Music Educators Journal, [s. l.], v. 54, n. 6, p. 72-171, 1968.

New introduction: Religions, Values and Peak-Experiences. Journal of Transpersonal Psychology, [s. l.], v. 2, n. 2, p. 83-90, 1970.

The farther reaches of human nature. London: Penguin, 1971.

MCCRAE, Robert Roger. The five-factor model of personality traits: consensus and controversy. In: CORR, P. J.; MATTHEWS, G. (ed.). Handbook of personality psychology. Cambridge: Cambridge University Press, 2009. p. 148-161.

NORTJÉ, Elsabe; VAN DER MERWE, Liesl. Young children and spirituality: understanding childrens connectedness in a group music class. International Journal of Children's Spirituality, [s. l.], v. 21, n. 1, p. 3-18, 2016.

PALMER, Anthony John. Music education and spirituality: a philosophical exploration II. Philosophy of Music Education Review, Bloomington, v. 14, n. 2, p. 143-158, 2006.

PARK, Nansook; PETERSON, Christopher; SELIGMAN, Martin E. P. Strengths of character and well-being. Journal of Social and Clinical Psychology, New York, v. 23, n. 5, p. 603-619, 2004.

PELLEGRINO, Kristen. Becoming music-making music teachers: connecting music making, identity, wellbeing, and teaching for four student teachers. Research Studies in Music Education, [s. l.], v. 37, n. 2, p. 175-194, 2015.

ROULSTON, Kathryn; JUTRAS, Peter; KIM, Seon Joo. Adult perspectives of learning musical instruments, International Journal of Music Education, [s. l.], v. 33, n. 3, p. 325-335, 2015. 
SENAY, Banu. Masterful words: musicianship and ethics in learning the ney. Journal of the Royal Anthropological Institute, [s. l.], v. 21, n. 3, p. 524-541, 2015.

SHEVOCK, Daniel J. Satis Coleman - a spiritual philosophy for music education. Music Educators Journal, [s. l.], v. 102, n. 1, p. 56-61, 2015.

SILVERMAN, Marissa. I drum, I sing, I dance: an ethnographic study of a West African drum and dance ensemble. Research Studies in Music Education, [s. l.], v. 40, n. 1, p. 5-27, 2018.

SOMMERMAN, Américo. Inter ou transdisciplinaridade?: da fragmentação disciplinar ao novo diálogo entre os saberes. 2. ed. São Paulo: Paulus, 2008.

SOUZA, Marcela Tavares; SILVA, Michelly Dias da; CARVALHO, Rachel de. Revisão integrativa: o que é e como fazer. Einstein, São Paulo, v. 8, n. 1, p. 102-106, 2010.

STRIEDER, Roque; HERBERT, Fabiana. Educação inclusiva: também um desafio de espiritualidade. Revista Educação Especial, Santa Maria, v. 30, n. 58, p. 405-424, 2017.

TECCHIO, Edivandro Luiz; CUNHA, Cristiano José Castro de Almeida; SANTOS, Fabiana Besen. Spirituality in organizations?. Organizações \& Sociedade, Salvador, v. 23, n. 79, p. 590-608, 2016.

TECCHIO, Edivandro Luiz; CUNHA, Cristiano José Castro de Almeida; BRAND, Josiane Luisa. Espiritualidade nas organizações e criação de conhecimento. Organizações em Contexto, São Bernardo do Campo, v. 14, n. 27, p. 397-426, 2018.

VAN DER MERWE, Liesl; HABRON, John. A conceptual model of spirituality in music education. Journal of Research in Music Education, [s. l.], v. 63, n. 1, p. 47-69, 2015.

VAN DER MERWE, Liesl; HABRON, John. The Dalcroze diamond: a theory of spiritual experiences in Dalcroze eurhythmics. Music Education Research, [s. l.], v. 21, n. 4, p. 426-440, 2019.

WHITTEMORE, Robin; KNAFL, Kathleen. The integrative review: updated methodology. Journal of Advanced Nursing, [s. l.], v. 52, n. 5, p. 546-553, 2005. 
Denise Maria Bezerra é mestra em Música/Práticas Interpretativas (Piano) pela Universidade do Estado de Santa Catarina (UFSC) e doutoranda pelo Programa de Pós-Graduação em Engenharia e Gestão do Conhecimento da Universidade Federal de Santa Catarina (PPGEGC), na área de Mídias do Conhecimento. Graduada em Comunicação Social pela UFSC, é especialista em Educação Musical pela Universidade do Estado de Santa Catarina (Udesc), em Psicopedagogia Clínica pela Universidade do Sul de Santa Catarina (Unisul) e em Psicologia Transpessoal pela Associação Luso-Brasileira de Transpessoal (Alubrat). Participa como membro do Núcleo de Estudos e Desenvolvimento em Conhecimento e Consciência (NEDEC2), ligado ao PPGEGC, onde criou o Laboratório de Cognição e Psicologia da Música (Lacomusi), com seu orientador Professor Dr. Francisco Antonio Pereira Fialho. Por sua vasta experiência clínica em psicopedagogia e música, realiza palestras e workshops sobre as temáticas de autoconhecimento, motivação, estratégias cognitivas e metacognitivas na prática pianística. https://orcid.org/0000-0002-7235-9456

Francisco Antonio Pereira Fialho é psicólogo, mestre e doutor em Engenharia de Produção e Engenharia do Conhecimento. Graduado em Engenharia Eletrônica, atualmente é professor titular da Universidade Federal de Santa Catarina (UFSC) no Programa de Pós-Graduação em Engenharia e Gestão do Conhecimento (PPGEGC). Lidera os grupos de pesquisa Núcleo de Estudos e Desenvolvimentos em Conhecimento e Consciência (NEDEC2) e Núcleo de Pesquisas em Complexidade e Cognição (Nucog), na UFSC. Possui mais de 200 artigos publicados em periódicos nacionais e internacionais. Autor de vários livros, entre eles Psicologia das atividades mentais: introdução às ciências da cognição (2011) e Uma escola para os magos do amanhã: um ser interdisciplinar aberto ao diálogo (2011). https://orcid.org/0000-0001-6826-7180 